\title{
Gonadal Hormone Trigger Traf6-mediated Dynamic Microglial Alterations That Correlate With Depressive Symptomatology
}

\section{Rui Peng ( $\sim$ pengruiwec@163.com )}

Renmin Hospital of Wuhan University: Wuhan University Renmin Hospital

\section{Wen Dai}

Renmin Hospital of Wuhan University: Wuhan University Renmin Hospital

Di Li

Renmin Hospital of Wuhan University: Wuhan University Renmin Hospital

Yan Li

Renmin Hospital of Wuhan University: Wuhan University Renmin Hospital

\section{Research Article}

Keywords: Gonadal hormone, Deficiency, Replacement, Microglia, Neuroimmune, Depression

Posted Date: December 28th, 2021

DOI: https://doi.org/10.21203/rs.3.rs-1130484/v1

License: (c) (i) This work is licensed under a Creative Commons Attribution 4.0 International License. Read Full License 


\section{Abstract}

Backgrounds: Gonadal hormone deficiency is associated with the development of depression, but what mediates this association is unclear. To test the possibility that it reflects neuroimmune and neuroinflammatory processes, we analyzed how gonadal hormone deficiency and replacement affect microglial activation and inflammatory response during the development of depressive symptomatology in gonadectomized male mice.

Methods: Adult male ICR mice received gonadectomy. Gonadal hormone levels, neuroinflammation, mciroglial activation and depressive behaviors were evaluated 7 days, 14 days, and 30 days later. Furthermore, the neuroprotective mechanism of treatment with testosterone and estradiol on depressive symptomatology were also observed.

Results: Testosterone level and the ratio of testosterone to estradiol in the serum and brain tissue of mice exposed to 3-35 days of chronic unpredictable stress were much lower than in control animals. Gonadal hormone sustained deficiency in gonadectomized mice and subsequent led to acute inflammation at day 7 following castration. Activating microglia in mice exposed to 7 days of castration subsequently suppressed the proliferation of microglia, such that their numbers in hippocampus and cortex were lower than the numbers in sham-operated mice after 30 days of castration. Here, we showed that gonadal hormone deficiency induces Traf6-mediated microglia activation, a type of inflammatory mediator. Microglia treated in this way for long time showed down-regulation of activation markers, abnormal morphology and depressive-like behaviors. Restoration and maintenance of a fixed ratio of testosterone to estradiol significantly suppressed microglial activation, neuronal necroptosis, dramatically inducing hippocampal neurogenesis and reducing depressive behaviors via the suppression of Traf6/TAK1 pathway.

Conclusions: These findings suggest that activated or immunoreactive microglia contribute to gonadal hormone deficiency-induced depression, as well as testosterone and estradiol exert synergistic antidepressant effects via suppressing microglial activaton in gonadectomized male mice, possibly through Traf6 signaling.

\section{Background}

Major depressive disorder is approximately 2-3 times as likely to happen in women as in men [1,2], indicating a crucial role for gonadal hormones in the pathogenesis of this disease. Gonadal hormone may contribute to this sex difference [3, 4]. Increased level of testosterone appears to protect against major depressive disorder and to reduce depressive behaviors in men and women [5-11]. In rodents, testosterone also works as an anti-depressant in older male mice [12], and it can decrease the incidence of depressive symptoms in rats [13]. It seems clear that testosterone can regulate diseases related to depression, but which brain cells it affects and how it affects them remain poorly understood. 
Changes in hippocampal levels of neurotrophic factors such as brain-derived neurotrophic factor (BDNF) and nerve growth factor (NGF) may contribute to the onset and treatment of major depressive disorder [14-18]. In fact, testosterone may reduce depressive behaviors by increasing BDNF expression and neurogenesis in hippocampus. Testosterone may also interact directly with the androgen receptor or undergo aromatization into estrogen metabolites in the brain to activate the mitogen-activated protein kinase (MAPK) signaling pathway [19-21], which mediates alterations in emotion and mood. Consistent with this idea, exogenous testosterone can reduce depressive behaviors by undergoing aromatization into estrogen metabolites in the dentate gyrus area of gonadectomized male rats [22].

Like testosterone, estradiol exerts anxiolytic and anti-depressant activity in gonadectomized male rats [22]. Estradiol reduces depressive behaviors in ovariectomized rats in part by suppressing indoleamine2,3-dioxygenase activity and increasing 5-hydroxytryptamine expression in hippocampus [23]. Estradiol and testosterone have been shown to act synergistically in the treatment of early-stage atherosclerosis [24]. Less understood is whether and how testosterone and estradiol act synergistically to affect depressive behaviors, such as in mitigating depression in gonadectomized male mice.

It seems likely that testosterone and estradiol exert their anti-depressant effects at least in part by reducing microglial activation and neuroinflammatory responses. Microglia, which account for roughly $10 \%$ of the total number of brain cells, are resident innate immune cells of the central nervous system that become activated in response to brain infection, injury and neurodegenerative disorders [25]. Microglial priming as well as immune response may trigger depression-related complications after traumatic brain injury [26]. Microglia are involved in synaptic alterations, repair of micro-damage and neurogenesis [27-31]. The tumor necrosis factor receptor-associated factor 6 (Traf6) has nonconventional ubiquitin E3 ligase activity and plays functions in tissue homoeostasis and immune response [32-34]. Previous studies have demonstrated that the activation of Traf 6 signaling could promote opioid-induced damage to microglia and neurological injury [35, 36]. Traf6 is an essential adaptor for IL-1R and NF-KB signaling activation, and further regulates inflammatory response and cell spoptosis. Traf6 expression in the spinal cord was reported to play an important role in neuroinflammation and neuropathic pain [37]. Estrogen inhibits Traf6 expression in human monocytes via estrogen receptor-alpha (ERa), and serum testosterone was found to show a negative association with Traf6 in patients with metastatic cancer $[37,38]$. Therefore, the potential for synergistic effects between testosterone and estradiol on microglial activation and neuroinflammation should be explored. The present study investigated the dynamic alterations in neuroimmune cells in male mice following gonadectomy. It also explored the protective, anti-depressant effects of rebalancing sex hormones.

\section{Materials And Methods}

\section{Animals}

Adult male ICR mice 6 weeks old were purchased from Beijing Vital River Laboratory Animal Technology (China) and housed five per cage at $23 \pm 2{ }^{\circ} \mathrm{C}$ on a 12-h light/dark cycle with standard food and water ad 
libitum. Animals were kept in the Animal Laboratory Center of Renmin Hospital of Wuhan University.

\section{Ethical consideration}

Animals used for our study were handled based on the rules and regulations for experimental animal management as stated in the Guide for the Care and Use of Laboratory Animals. The present study was approved by Medical Ethics Review Committee of Renmin Hospital, Wuhan University, and an approval number S0271902190A assigned to the research.

\section{Stress exposure}

Animals were exposed to chronic unpredictable stress (CUS) as described [39], with minor modifications. For five weeks, animals were exposed daily to two of the following stressors in random order: forced swimming for $4 \mathrm{~min}$ in water at $40{ }^{\circ} \mathrm{C}$, forced swimming for 2 min in water at $4{ }^{\circ} \mathrm{C}$, sitting in a wet cage for $24 \mathrm{~h}$, tail-squeezing lasting $1 \mathrm{~min}$, crowding for $2 \mathrm{~h}$, food deprivation for $24 \mathrm{~h}$, and water deprivation for $24 \mathrm{~h}$. Shorter periods of unpredictable stress consisted of various combinations of three of the above mentioned stressors applied during 3 days.

\section{Gonadectomy and hormone replacement}

Mice were anesthetized by intraperitoneal injection of $1 \%$ pentobarbital sodium $(0.1 \mathrm{~mL} / 10 \mathrm{~g})$. Then a ventral midline incision $(1-2 \mathrm{~cm})$ was made in the scrotum to expose the tunica. The tunica was cut off and both testes and epididymis were pulled up to expose the cryptic vessels. These blood vessels were ligated with stylolites, and testes and epididymis were excised. Both vessels and adipose tissue were returned into the tunica, then the incision was sutured. Sham-operated animals were subjected to the same surgery, but the testes and epididymis were not excised, nor were blood vessels ligated. Animals subjected to gonadectomy or sham surgery were then assigned to one of three hormone replacement groups, which received intraperitoneal injections of $\beta$-estradiol (Sigma, Israel) alone at a dose of $1 \mu \mathrm{g} / \mathrm{d}$, testosterone (Aladdin, China) alone at a dose of $35 \mu \mathrm{g} / \mathrm{d}$, or testosterone and $\beta$-estradiol in a 35:1 ratio. Injections were given daily beginning 1 day after surgery and continuing for either one or five weeks after gonadectomy.

\section{Behavioral assays}

In the sucrose preference test (SPT), animals were allowed to habituate to sucrose for 3 days, then given access for $4 \mathrm{~h}$ to two drinking tubes (one containing water and the other $1 \%$ sucrose) at the beginning of the dark circadian phase. Anhedonia was assessed in terms of sucrose preference, which was calculated as the ratio of sucrose volume consumed to total drinking volume.

In the forced swim test (FST), mice were placed in a large glass cylinder $(30 \times 18 \mathrm{~cm})$ filled with water at 23-25 ${ }^{\circ} \mathrm{C}$ and allowed to habituate for $2 \mathrm{~min}$. Then animals were forced to swim for $4 \mathrm{~min}$, and the time spent immobile (moving only to remain afloat) was recorded [40]. The water in the cylinders was replaced between animals. 
The tail suspension test (TST) was carried out as described with minor adjustments [41]. Each mouse was suspended by the tail, and the time spent immobile (no movements other than whiskers or respiration) during 4 min was calculated.

\section{Microglia isolation}

Tissue in hippocampus and prefrontal cortex were collected to homogenized in HBSS, pH 7.4, by passing through a $70 \mu \mathrm{m}$ nylon cell strainer. Then homogenates were centrifuged at $700 \mathrm{~g} / \mathrm{min}$ for $5 \mathrm{~min}$. Cell pellets were recovered and resuspended in $70 \%$ isotonic Percoll at room temperature. A discontinuous Percoll density gradient was layered as follows: $70 \%, 50 \%, 35 \%$, and $0 \%$ isotonic Percoll. The Percoll was centrifuged at $2000 \mathrm{~g} / \mathrm{min}$ for $25 \mathrm{~min}$ and microglia were recovered from the interphase between the $70 \%$ and $50 \%$ Percoll layers. The purity of microglia in extractant more than $90 \%$.

\section{Western blotting}

Total protein was extracted from microglia, and Western blotting was used to determine levels of the inflammatory cytokines interleukin-6 (IL-6) and tumor necrosis factor-a (TNF-a), the apoptotic proteins (Bax and Bcl-2), related proteins in Traf6/TAK1 signaling (Traf6, TAK1 and p-TAK1) as well as the microglial activation markers anti-ionized calcium-binding adapter molecule-1 (Iba-1) and major histocompatibility complex protein II (MHC-II) [42]. Briefly, immune printing strips were incubated overnight at $4{ }^{\circ} \mathrm{C}$ with antibodies against IL-6 (1:1000; Santa Cruz Biotechnology), TNF-a (1:2000; Santa Cruz Biotechnology), Bax (1:1000; Cell Signaling Technology), Bcl-2 (1:1000; Abcam Biotechnology, UK), Traf6 (1:1000; Abcam Biotechnology, UK), TAK1 (1:1000; Abcam Biotechnology, UK), p-TAK1 (1:1000; Abcam Biotechnology, UK), Iba-1 (1:1000; Abcam Biotechnology, UK), or MHC-II (1:1000; Absin Bioscience, Shanghai; China). Blots were washed and incubated with horseradish peroxidase-conjugated goat antirabbit IgG (1:3000; Google Biological Technology, Wuhan, China). Blots were then analyzed using a chemiluminescence detection system (Thermo Scientific, USA).

\section{Enzyme-linked immunosorbent assay (ELISA)}

Hippocampal neurogenesis was measured by assaying levels of BDNF and NGF using commercial ELISA kits (Cusabio Biotech, China) according to the manufacturer's instructions. The manufacturer-specified detection limits were $15.6 \mathrm{pg} / \mathrm{ml}$ for BDNF and $1.56 \mathrm{pg} / \mathrm{ml}$ for NGF.

\section{LC-MS/MS assay of sex hormone in serum and brain}

Endogenous sex hormones were extracted from brain tissue/serum samples using liquid-liquid extraction. An aliquot $(100 \mu \mathrm{l})$ of tissue homogenate or serum was mixed with $10 \mu \mathrm{l}$ of internal standard and $500 \mu \mathrm{l}$ of methyl tert-butyl ether (MTBE), the mixture was vortexed for $5 \mathrm{~min}$, centrifuged at $6000 \mathrm{~g}$ for $10 \mathrm{~min}$, then the upper organic layer was transferred to a clean tube and dried under a nitrogen stream at $40{ }^{\circ} \mathrm{C}$. The residue was redissolved in $10 \mu \mathrm{l}$ of sodium bicarbonate $(0.1 \mathrm{M})$ and $10 \mu \mathrm{l}$ of dansyl chloride in acetone $(1 \mathrm{mg} / \mathrm{ml})$, vortexed for $30 \mathrm{~s}$ and incubated at $60^{\circ} \mathrm{C}$ for $10 \mathrm{~min}$. An aliquot $(20 \mu \mathrm{l})$ of the resulting dansyl chloride derivative of estrogen was purified on an Ekspert ultraLC 100-XL liquid chromatography system (Sciex, eksigent-AB, USA). Eluate was ionized using electrospray ionization (ESI) in positive mode 
at $550{ }^{\circ} \mathrm{C}$ and detected on-line with an AB SCIEX 4500 QTRAP mass spectrometer (Applied Biosystems, Foster City, CA). Area under the curve (AUC) was calculated from chromatograms for testosterone, testosterone-d3, estradiol, and estradiol-d2. Amounts of testosterone and estradiol in samples were determined by comparing the AUCs to curves of calibration standards chromatographed under the same conditions.

\section{Histology}

Whole blood was collected from the orbit at $12 \mathrm{~h}$ after the last behavioral test. Mice were then perfused through the left ventricle with ice-cold PBS for $5 \mathrm{~min}$, followed by $4 \%$ paraformaldehyde for $15 \mathrm{~min}$. The brain was quickly removed and hippocampus and perfrontal cortex were dissected on ice and placed in $4 \%$ paraformaldehyde as soon as possible. After $24 \mathrm{~h}$, the tissues were embedded in 5 - $\mu \mathrm{m}$ paraffin medium at $67{ }^{\circ} \mathrm{C}$ according to routine procedures. Sections in $4 \%$ paraformaldehyde were washed three times, then incubated overnight at $4{ }^{\circ} \mathrm{C}$ with rabbit antibody against Iba- 1 (1:300, Abcam, UK). Sections were incubated at room temperature for $1.5 \mathrm{~h}$ with goat biotinylated anti-rabbit IgG (1:200, Cell Signaling Technology, USA) and counter stained with DAPI (Abcam, UK). For Iba-1/Traf6 double-staining, Iba-1 staining was performed first according to the description above, and then followed by immunistaining of Traf6.

Images of Iba-1 fluorescence were captured using a fluorescence microscope (Nikon Eclipse TI-SR, Japan). The number of Iba-1-labeled microglia and area of fraction were analyzed using Image $\mathrm{J}$. Microglial body size and process length were evaluated using Metamorph Image Offline 6.1 (Molecular Devices, USA). Numbers of apoptotic cells in the hippocampus were determined using a commercial TUNEL kit (Roche Diagnostics, Indianapolis, IN, USA) according to the manufacturer's instructions.

\section{Statistical analyses}

All data are expressed as mean \pm s.d. All statistical differences were assessed for significance using one-, two-, or three-way analyses of variance after Tukey's post-hoc analyses or Bonferroni correction.

Differences between mean values were assessed using Student's $t$ test, when appropriate. All analyses were performed using SPSS 20.0 (IBM, Chicago, IL, USA).

\section{Results}

\section{Gonadal hormone imbalance in an animal model of depression}

To investigate the potential role of gonadal hormone in the development of depressive disorder, we first examined whether levels of testosterone and estradiol as well as the ratio of testosterone/estradiol were altered in male depressive mice relative to control subjects. Our results indicate that between 3 and 35 days after chronic unpredictable stress, testosterone levels in serum and brain tissue were lower in stressed mice than in controls. Serum levels were 0.89 -fold relative to control at 3 days, 0.54 -fold at 14 
days and 0.36 -fold at 35 days. Tissue levels were 0.72 -fold at 3 days, 0.40 -fold at 14 days and 0.34 -fold at 35 days (Figure $1 \mathrm{~A}$ and $\mathrm{C}$ ). Similar results were observed for the ratio of testosterone/estradiol: the ratio in serum in stressed animals was 0.85 -fold relative to control mice at 3 days, 0.56 -fold at 14 days and 0.40 -fold at 35 days; the ratio in brain tissue in stressed mice was 0.73 -fold relative to control mice at 3 days, 0.54 -fold at 14 days, and 0.45 -fold at 35 days (Figure 1D and F). Over the same period after chronic unpredictable stress, estradiol levels in serum and brain tissue did not differ substantially between stressed and control animals (Figure 1B and E). These results suggest that gonadal hormone imbalance in the form of reduced testosterone and testosterone/estradiol ratio in serum and brain may contribute to depression.

\section{Effects of short-term gonadal hormone deficiency on neuroinflammation and microglial priming}

Gonadal hormone central to immune function, exerting anti-inflammatory effects on multiple tissues and organs. Therefore, we determined the effects of short-term gonadal hormone deficiency on neuroinflammation and microglial activation. We found that the levels of two inflammatory cytokines associated with microglial activation, IL-6 and TNF-a, were significantly higher in the cortical and hippocampal microglia of gonadectomized mice than in sham-operated controls at day 7 following gonadectomy (Figure 2E-G). By day 14, levels of both cytokines in microglia of gonadectomized mice had fallen below the levels in sham-operated controls. These results suggest that gonadal hormone deficiency can trigger a periodic inflammatory response in microglia of cortex and hippocampus.

Consistent with these effects on neuroinflammatory responses, gonadal hormone deficiency affected microglial activation. On day 7 following gonadectomy, Iba-1 immunoreactivity in hippocampal microglia was much higher than in sham-operated animals (Figure 2B), and microglia showed an activated morphology with shorter microglial processes (Figure 2C) and larger somata (Figure 2D). By day 14 following gonadectomy, lba-1 immunoreactivity and soma size had returned to baseline, whereas microglial processes were still significantly shorter than in sham-operated mice (Figure 2A-D).

\section{Effects of long-term gonadal hormone deficiency on microglial decline as well as depressive behavior}

To investigate whether depression linked to gonadal hormone deficiency involves changes in microglia, we gonadectomized mice and 30 days later, evaluated depressive symptomatology as well as the number and morphology of microglia (Figure 3A). Gonadectomy was associated with significantly longer immobile time in the FST and TST, as well as decreased sucrose preference (Figure 3B-D). It was also associated with markedly reduced Iba-1 immunoreactivity in hippocampus but not in cortex (Figure 4A-D), as well as dystrophic morphology of some microglia within both hippocampus and cortex, observed as shorter microglial processes (Figure 4C) and smaller somata (Figure 4D).

At the same time, gonadectomy was associated with significantly lower expression of the microglial activation markers MHC- $₫$ and Iba- 1 in the hippocampus (Figure 4E and F). These results suggest that the 
development of depressive symptomatology is associated with suppression of microglia.

\section{Gonadal hormone deficiency induces Traf6-mediated microglial activation}

To observe whether Traf 6 contributes to gonadal hormone deficiency-induced microglial activation, we then determined the Traf6-positive microglia in hippocampus of sham-operated and gonadectomized mice using Iba-1/Traf6 double-staining. We found that the number of Traf6-positive microglia were significantly increased in the hippocampus of gonadectomized mice compared with sham-operated controls at day 7 following gonadectomy (Figure 5A). However, the number of Traf6-positive microglia at day 14 and 30 had fallen compared to day 7 . Western Blotting demonstrated that the expression levels of Traf6 and p-TAK1 in hippocampal microglia of mice exposed to 7 days of castration were significantly increased compared to sham-operated controls, while these levels showed a decreasing trend at day 14 and 30 (Figure 5B-D).

\section{Effect of sex hormone replacement in gonadectomized mice on microglial priming}

To explore the impacts of sex hormone replacement on the microglial activation in the hippocampus of male gonadectomized mice, gonadectomized mice were treated with hormone replacement in the form estradiol alone $(E)$, testosterone $(T)$ alone or the mixture of testosterone and estradiol in a 35:1 ratio (T/E) for 7 days. Our previous work has demonstrated that the mixture of testosterone and estradiol in a 10:1 ratio could synergistically suppress apoptosis in PC12 cells with corticosterone-induced injury (43). And this study also showed that a 35:1 ratio of testosterone and estradiol synergetic regulates the activation of microglia in hippocampus of male gonadectomized mice. The expression levels of the microglial activation markers MHC-II and Iba-1 were return to baseline levels similar to those of sham-operated animals when treated with a fixed testosterone/estradiol ratio, while the similar impacts were not found in the treatment with testosterone and estradiol alone (Figure 6A-C). Furthermore, the similar result that Iba1 immunoreactivity in hippocampal microglia of gonadectomized mice treated with a defined testosterone/estradiol ratio was much lower than those of gonadectomized mice treated with testosterone and estradiol alone was found (Figure 6D and E). These findings suggested that an appropriate dosage of testosterone combined with estradiol may exert a synergistic effect for blocking the microglial priming.

\section{Effect of gonadal hormone replacement in gonadectomized mice on neuronal apoptosis}

Some researchers have indicated that microglial priming subsequently induced the neuronal apoptosis via activating the proinflammatory signaling [44]. Therefore, we observed the effect of gonadal hormone replacement in gonadectomized mice on neuronal apoptosis by determining the apoptotic proteins and TUNEL-stained cells in the hippocampus (Figure 7A). The expressions of $\mathrm{Bcl}-2$ and $\mathrm{Bax}$ proteins were returned to baseline levels similar to those of sham-operated mice when supplemented with a defined 
testosterone/estradiol ratio, whereas the similar role was not investigated in the supplementation with testosterone and estradiol alone (Figure 7B and C). Moreover, the similar result of TUNEL staining was also observed (Figure 7D and E), indicating that an appropriate ratio of testosterone combined with estradiol may have a synergistic role in protecting the neurons against apoptosis.

\section{Effects of hormone replacement on depressive behavior, neuroinflammatory signaling and neurogenesis}

The experiments described above suggested that sex hormone imbalance was associated with altered neuroimmune function and development of depressive behaviors. This prompted us to ask whether restoring sex hormone balance would reverse the effects of gonadectomy. Therefore, we treated gonadectomized mice with testosterone alone, estradiol alone or both hormones in a ratio of 35:1 to observe the role of sex hormone replacement in depressive symptomatology (Figure 8A).

To explore the effect of hormone replacement on neurogenesis, we used ELISA assay to measure the contents of nerve growth factor (NGF) and brain derived neurotrophic factor (BDNF) in the hippocampus. Only BDNF levels were higher following the replacement of testosterone alone or both hormones in a ratio of $35: 1$ by 5 weeks after gonadectomy (Figure 8D). The duration of this response differed among the three treatments: in animals given the 35:1 ratio of both sex hormones, the level of BDNF returned to levels similar to those in sham mice; this result was not observed in animals given testosterone or estradiol alone. However, this therapy did not affect levels of NGF (Figure 8D). Furthermore, all three replacement treatments reversed gonadectomy-induced changes in sucrose preference (Figure 8G) and in performance on the FST (Figure 8E) and TST (Figure 8F). In addition, the synergistic protective effects of the defined testosterone/estradiol ratio on depressive-like acts were also investigated according to comparing depressive behavior in three types of replacement treatments.

Our previous work has elucidated that testosterone/estradiol synergetic suppress Traf6 expression mainly via androgen receptor (AR)/estrogen receptor a (ERa)-mediated pathways [43]. Further study showed that AR and ERa directly interact with Traf6 and suppress its ubiquitination. This research suggested that all three treatments led to much smaller gonadectomy-induced increases in IL- 6 and TNFa levels via partly blocking Traf6/TAK1 signaling (Figure 8B and C). In addition, the synergistic antiinflammatory effect of the defined testosterone/estradiol ratio on the hippocampus was also observed according to comparing inflammatory cytokine levels. Collectively, testosterone/estradiol synergetic inhibit inflammatory response through decreasing Traf6 expression to suppress the microglial activation.

\section{Discussion}

Our findings suggest that gonadal hormone deficiency causes an inflammatory response, followed initially by microgial proliferation and activation, and later by microglial dystrophy and decline. These effects preferentially target the hippocampus and lead to a shrinking of the microglia population as well as smaller microglia somata. These effects may therefore contribute to the observed decline of hippocampal volume in depressive patients [45]. We found that hormone replacement at a 
testosterone/estradiol ratio of 35:1 suppressed inflammation and microglial activation in gonadectomized mice and reduced depressive behavior. These findings suggest a model in which an over-expression of Traf6 contributes to gonadal hormone deficiency-induced microglial activation or immunoreaction leading to the development of depressive-like behaviors.

We found that short-term gonadectomy induced the activation of Traf6/TAK1 signaling, corresponding to inflammatory response as reported in previous work $[46,47]$. At the same time, short-term gonadectomy stimulated inflammation, similar to previous results [48], and subsequently triggered microglial proliferation and activation. Consistent with our results, previous work showed that exposing mice to lipopolysaccharide activates microglia within $6 \mathrm{~h}$ [49], exposing animals to live Escherichia coli activates microglia after 2 days [50], and exposing leptomeningeal cells to heat-killed bacteria activates microglia after 2 weeks [51]. These results indicate that gonadal hormone deficiency compromises the blood-brain barrier [52], triggering the release of peripheral inflammatory cytokines into the brain followed by microglial proliferation and activation. The role of inflammatory cytokines in activating microglia and stimulating their proliferation is supported by our observation that hormone replacement with the 35:1 testosterone/estrogen ratio reversed the microglial activation induced by gonadectomy. This initial activation and proliferation of microglia subsequently leads to microglial decline, decreased synaptic remodeling, suppressed neurogenesis, and depressive behaviors, which we were able to reverse in our study through hormone replacement with the testosterone/estrogen ratio. Our work is consistent with several previous studies documenting the neuroprotective effects of androgens and estrogen specifically [53-58]. In all these cases, it appears that the sex hormone inhibits microglial proliferation and activation, albeit through different mechanisms.

Earlier studies in rodents have suggested that pathogenic bacteria, aging, brain injury, and gonadal hormone deficiency stimulate an immunoreactive population of microglia [59-62], whose activation can be observed as increased Iba-1 and MHC-II staining, shortening of cell processes, increase in cell soma area, and acute production of inflammatory cytokine after immunosuppression. The present study extends that literature by showing that gonadal hormone deficiency leads to a large number of activated microglia that develop and persist in the hippocampus for at least 7 days after gonadectomy. Interestingly, by day 14 , microglial Iba-1 staining and soma area had returned to baseline, although cell processes remained shorter. This time-dependent effect echoes a previous study in which unpredictable stress caused microglia in the dentate gyrus to become hyperactive on day 2, yet by day 4 their activation status had reverted to baseline [63]. In the present study, we found that at 30 days after gonadectomy, microglial apoptosis increased and astrocyte number fell, followed by onset of depressive behaviors. Our results demonstrate that using microglial inhibitors to block microglial activation and subsequent neuronal apoptosis can alleviate depressive behaviors. In support of this notion, 1-week gonadal hormone replacement inhibited microglial activation, and 5-week replacement caused gonadectomyinduced neuroinflammation, neurogenesis suppression, neuronal apoptosis and depressive behavior to revert to baseline. 
Studies suggest that microglia are not passive, simply waiting to be activated by various stimulus, but rather that they are active even when "at rest": they interact with neurons and astrocytes via tight intercellular junctions to regulate physiological processes such as nutrient transport and synaptic formation [64-66]. Our results suggest that depressed symptomatology associated with gonadal hormone deficiency is related to microglial suppression, rather than activation. Thus, analysis at 30 days after gonadectomy showed microglial decline, increased immobile time in the FST and TST, and decreased sucrose preference. Gonadal hormone deficiency was also associated with elevated levels of the neuroinflammatory factors IL- 6 and TNF-a.

Our observations of synergism between testosterone and estradiol in the development of depression and in their therapeutic effects is consistent with our previous report that the 10:1 ratio of testosterone/estradiol synergistically inhibits apoptosis in PC12 cells via the inhibition of Traf6/TAK1 signaling (43). Traf6 regulates TLRs-mediated pro-inflammatory cytokine production [67]. This study also demonstrated that the 35:1 ratio of testosterone/estradiol synergistically suppresses the microglial priming via decreasing Traf 6 expression and the levels of inflammatory cytokines, suggesting that Traf 6 is a key mediator for regulating the activation of microglia in gonadectomized mice. Similar synergistic therapeutic effect has been reported in early-stage atherosclerosis, proceptive behavior, prostatic hyperplasia, and abnormal spermatogenesis [15, 68-69]. Our results suggest that this synergism exerts anti-depressant effects at least in part by inhibiting microglial activation. In contrast, testosterone on its own has previously been shown to exert anti-depressant effects by undergoing aromatization, and then the resulting derivative induces over-expression of extracellular signal-regulated kinase 2 (ERK2) within the dentate gyrus $[14,23]$. Our findings appear to be linked to suppression of hippocampal inflammatory cytokines, and induction of neurogenesis.

The neuroprotective roles of testosterone and estradiol were well demonstrated in previous publications according to clinical retrospective studies and depressive model investigations [5-14, 70]. Although the molecular mechanisms of depression remain unclear, the pathophysiology that excessive induction of the hypothalamic-pituitary-adrenal cortical (HPA) axis leading to increased glucocorticoid abundances and decreased feedback suppression of the axis [71]. Moreover, our findings display that the pathophysiology, gonadal hormone deficiency, results in microglial activation in hippocampus followed by the development of depression. Through the microglail activation, subsequent excess decline of microglia may contribute to sex hormone deficiency-induced structural alterations in the hippocampus $[72,73]$, a phenomenon often related to the decreased releasing of BDNF. The neurotrophic theory of depression refers to the decreased of hippocampal BDNF level, which can be ameliorated through the treatment with antidepressants and replacements of gonadal hormone in our study [74]. Accordingly, it is likely that testosterone and estradiol can induce the expression level of BDNF. Testosterone and estradiol were demonstrated to have neuroprotective roles through different molecular mechanisms, respectively [21-24], whereas our findings show that they have a significant synergistic antidepressant-like effects in gonadectomized male mice via suppressing the microglial activation. However, the molecular mechanisms in suppressed microglial after treatment with sex hormone need to be further studied. 


\section{Conclusion}

Our study provides the first detailed evidence that gonadal hormone deficiency hyperactivates microglia in the hippocampus, which is associated with development of depressive behaviors. We further show that replacement therapy with testosterone and estradiol in a well-defined ratio can exert synergistic antidepressant effects via inhibiting Traf6/TAK1 cascade activation. They imply that individuals with lower gonadal hormone levels are more sensitive to neuroinflammation followed by microglial activation. They also imply that creating an appropriate testosterone/estradiol ratio can inhibit microglia and thereby block the development of depressive behaviors.

\section{Abbreviations}

BDNF: brain-derived neurotrophic factor; NGF: nerve growth factor; MAPK: mitogen-activated protein kinase; Traf6: tumor necrosis factor receptor-associated factor 6; ERa: estrogen receptor-alpha.

\section{Declarations}

\section{Authors' contributions}

RP carried out literature searches, prepared figures, did data interpretation and writing; DL did writing and editing; $Y L$ and WD conceptualized the paper, prepared figures, did writing and editing. The authors read and approved the final manuscript.

\section{Acknowledgments}

We thanks the Renmin Hospital of Wuhan University to provided for the technical consultation.

\section{Competing interests}

The authors declare that they have no competing interests.

\section{Availability of data and materials}

Please contact author for data requests.

\section{Funding}

This study was supported by the Fundamental Research Funds for the Central Universities (No. 2042020kf0064).

\section{References}

1. Bebbington P, Dunn G, Jenkins R. The influence of age and sex on the prevalence of depressive conditions: Report from the National Survey of Psychiatric Morbidity. Int Rev Psychiatry. 2003; 15: 
74-83.

2. Kessler RC. Epidemiology of women and depression. Journal of Affective Disorders. 2003; 74: 5-13.

3. Becker JB, Monteggia LM, Perrot-Sinal TS. Stress and disease: is being female a predisposing factor. J Neurosci. 2007; 27: 11851-11855.

4. Dalla C, Antoniou K, Kokras N. Sex differences in the effects of two stress paradigms on dopaminergicneurotransmission. Physiol Behav. 2008; 93: 595-605.

5. Kanayama G, Amiaz R, Seidman S. Testosterone supplementation for depressed men: current research and suggested treatment guidelines. Exp Clin Psychopharmacol. 2007; 15: 529-538.

6. Mclntyre RS, Mancini D, Eisfeld BS,. Calculated bioavailable testosterone levels and depression in middle-aged men. Psychoneuroendocrinology. 2006; 31: 1029 -1035.

7. Cunningham GR, Cordero E, Thornby JI. Testosterone replacement with transdermal therapeutic systems. Physiological serum testosterone and elevated dihydrotestosterone levels. JAMA. 1989; 261: 2525-2530.

8. McNicholas TA, Dean JD, Mulder H. A novel testosterone gel formulation normalizes androgen levels in hypogonadal men, with improvements in body composition and sexual function. BJU Int. 2003; 91: 69-74.

9. Vermeulen A, \& Kaufman JM. Diagnosis of hypogonadism in the aging male. Aging Male. 2002; 5: 170-176.

10. Snyder PJ, Bhasin S, Cunningham GR, et al. Effects of testosterone treatment in older men. $\mathrm{N}$ Engl $\mathrm{J}$ Med. 2016, 374: 611-624.

11. Shifren JL, Braunstein GD, Simon JA. Transdermal testosterone treatment in women with impaired sexual function after oophorectomy. N Engl J Med. 2000; 343: 682-8.

12. Frye CA, Walf AA. Depression-like behavior of aged male and female mice is ameliorated with administration of testosterone or its metabolites. Physiol Behav. 2009; 97: 266-269.

13. Solomon MB, Karom MC, Norvelle A. Gonadal hormones modulate the display of conditioned defeat in male Syrian hamsters. Horm Behav. 2009; 56: 423-428.

14. Krishnan V, Nestler EJ. The molecular neurobiology of depression.Nature. 2008; 455: 894 -902.

15. Perera TD, Coplan JD, Lisanby SH. Antidepressant-induced neurogenesis in the hippocampus of adult nonhuman primates. J Neurosci. 2007; 27: 4894-4901.

16. Madsen TM, Treschow A, Bengzon J. Increased neurogenesis in a model of electroconvulsive therapy. Biol Psychiatry. 2000; 47: 1043-1049.

17. Santarelli L, Saxe M, Gross C. Requirement of hippocampal neurogenesis for the behavioral effects of antidepressants. Science. 2003; 301: 805- 809.

18. Warner-Schmidt JL, Duman RS. Hippocampal neurogenesis: Opposing effects of stress and antidepressant treatment. Hippocampus. 2006; 16: 239-249.

19. Migliaccio A, Castoria G, Di Domenico M. Steroid-induced androgen receptor-oestradiol receptor betaSrc complex triggers prostate cancer cell proliferation. EMBO J. 2000; 19: 5406-5417. 
20. Trentani A, Kuipers SD, Ter Horst GJ. Selective chronic stress-induced in vivo ERK1/2 hyperphosphorylation in medial prefrontocortical dendrites: implications for stress-related cortical pathology? Eur J Neurosci. 2002; 15: 1681-91.

21. Carrier N, Kabbaj M. Extracellular signal-regulated kinase 2 signaling in the hippocampal dentate gyrus mediates the antidepressant effects of testosterone. Biol Psychiatry. 2012; 71: 642-651.

22. Carrier N, Saland SK, Duclot F. The anxiolytic and antidepressant-like efects of testosterone and estrogen in gonadectomized male rats. Biol Psychiatry. 2015; 78: 259-269.

23. Xu Y, Sheng H, Tang Z. Inflammation and increased IDO in hippocampus contribute to depressionlike behavior induced by estrogen deficiency. Behav Brain Res. 2015; 288: 71-78.

24. Dai W, Ming W, Li Y. Synergistic effect of a physiological ratio of estradiol and testosterone in the treatment of early-stage atherosclerosis. Arch Med Res. 2015; 46: 619-29.

25. Prinz M, Priller J. Microglia and brain macrophages in the molecular age: from origin to neuropsychiatric disease. Nat Rev Neurosci. 2014; 15: 300-312.

26. Tremblay ME, Stevens B, Sierra A. The role of microglia in the healthy brain. J Neurosci. 2011; 31: 16064-69.

27. Kettenmann H, Hanisch UK, Noda M. Physiology of microglia. Physiol Rev. 2011; 91: 461-553.

28. Dantzer R, O'Connor JC, Freund GG. From inflammation to sickness and depression: when the immune system subjugates the brain. Nat Rev Neurosci. 2008; 9: 46-56.

29. Ekdahl CT, Kokaia Z, Lindvall O. Brain inflammation and adult neurogenesis: the dual role of microglia. Neuroscience. 2009; 158: 1021-1029.

30. Santarelli L, Saxe M, Gross C. Requirement of hippocampal neurogenesis for the behavioral effects of antidepressants. Science. 2003; 301: 805-809.

31. Fenn AM, Gensel JC, Huang Y. Immune activation promotes depression 1 month after diffuse brain injury: a role for primedmicroglia. Biol Psychiatry. 2014; 76: 575-584.

32. Ivanova SA, Semke VY, Vetlugina TP. Signs of apoptosis of immunocompetent cells in patients with depression. Neurosci Behav Physiol. 2007; 37: 527-530.

33. Wolf SA, Steiner B, Akpinarli A. CD4-positive T lymphocytes provide a ueuroimmunological link in the control of adulthippocampal neurogenesis. J Immunol. 2009; 182: 3979-84.

34. Guan SZ, Liu JW, Fang EF. Chronic unpredictable mild stress impairs erythrocyte immune function and changes T-lymphocyte subsets in a rat model of stress-induced depression. Environ Toxicol Pharmacol. 2014; 37: 414-422.

35. Atallah A, Mhaouty-Kodja S, Grange-Messent V. Chronic depletion of gonadal testosterone leads to blood-brain barrier dysfunction and inflammation in male mice. J Cereb Blood Flow Metab. 2017; 37: 3161-3175.

36. Barreto G, Veiga S, Azcoitia I. Testosterone decreases reactive astroglia and reactive microglia after brain injury in male rats: role of its metabolites, oestradiol and dihydrotestosterone. Eur J Neurosci. 2007; 25: 3039-3046. 
37. Kissick HT, Sanda MG, Dunn LK. Androgens alter T-cell immunity by inhibiting T-helper 1 differentiation. Proc Natl Acad Sci USA. 2014; 111: 9887-9892.

38. Dogan E, Erkoc R, Demir C. Effect of hormone replacement therapy on $\mathrm{CD} 4^{+}$and $\mathrm{CD} 8^{+}$numbers, $\mathrm{CD}^{+} / \mathrm{CD}^{+}$ratio, and immunoglobulin levels in hemodialysis patients. Renal Failure. $2005 ; 27: 421-$ 424.

39. Kreisel T, Frank MG, Licht T. Dynamic microglial alterations underlie stress-induced depressive-like behavior and suppressed neurogenesis. Mol Psychiatry. 2014;19: 699-709.

40. Lucki I. The forced swimming test as a model for core and component behavioral effects of antidepressant drugs. Behav Pharmacol. 1997; 8: 523-532.

41. Corona AW, Norden DM, Skendelas JP. Indoleamine 2,3-dioxygenase inhibition attenuates lipopolysaccharide induced persistentmicroglial activation and depressive-like complications in fractalkine receptor (CX(3)CR1)-deficientmice. Brain Behav Immun. 2013; 31: 134-42.

42. Dai W, Ming W, Li Y. Synergistic effect of a physiological ratio of estradiol and testosterone in the treatment of early-stage atherosclerosis. Arch Med Res. 2015; 46: 619-629.

43. Peng R, Dai W, Li Y. Neuroprotective effect of a physiological ratio of testosterone and estradiol on corticosterone- induced apoptosis in PC12 cells via Traf6/TAK1 pathway. Toxicol In Vitr. 2018; 50: 257-263.

44. Stratmann M, Konrad C, Kugel $\mathrm{H}$. Insular and hippocampal gray matter volume reductions in patients with major depressive disorder. PLoS One. 2014; 9:

45. McKinnon MC, Yucel K, Nazarov A. A meta-analysis examining clinical predictors of hippocampal volume in patients with major depressive disorder. J Psychiatry Neurosci. 2009; 34: 41-54.

46. Aboudkhil S, Bureau JP, Garrelly L. Effects of castration, depo-testosterone and cyproterone acetate on lymphocyte T subsets in mouse thymus and spleen. Scand J Immunol. 1991; 34: 647-53.

47. Page ST, Plymate SR, Bremner WJ. Effect of medical castration on CD4CD25 T cells, CD8 T cell IFNexpression, and NK cells: a physiological role for testosterone and/or its metabolites. Am J Physiol Endocrinol Metab. 2006; 290: E856-E863.

48. Tsilidis KK, Rohrmann S, McGlynn KA. Association between endogenous sex steroid hormones and inflammatory biomarkers in US men. Andrology. 2013; 1: 919-28.

49. Chen Z, Jalabi W, Shpargel KB. Lipopolysaccharide-induced microglial activation and neuroprotection against experimental braininjury is independent of hematogenous TLR4. J Neurosci. 2012; 32: 11706-11715.

50. Hoogland IC, Houbolt C, van Westerloo DJ. Systemic inflammation and microglial activation: systematic review of animal experiments. J Neuroinflammation. 2015; 12: 114-123.

51. Wu Z, Zhang J, Nakanishi H. Leptomeningeal cells activate microglia and astrocytes to induce IL-10 production by releasing pro-inflammatory cytokines during systemic inflammation. J Neuroimmunol. 2005; 167: 90-98. 
52. Atallah A, Mhaouty-Kodja S, Grange-Messent V. Chronic depletion of gonadal testosterone leads to blood-brain barrier dysfunction and inflammation in male mice. J Cereb Blood Flow Metab. 2017; 37: 3161-3175.

53. Arevalo MA, Santos-Galindo M, Acaz-Fonseca E. Gonadal hormones and the control of reactive gliosis. Horm Behav. 2013; 63: 216-221.

54. Brown $\mathrm{CM}, \mathrm{Xu} \mathrm{Q}$, Okhubo N. Androgen-mediated immune function is altered by the apolipoprotein $\mathrm{E}$ gene. Endocrinology. 2007; 148: 3383-3390.

55. Colton CA, Brown CM, Vitek MP. Sex steroids, APOE genotype and the innate immune system. Neurobiol Aging. 2005; 26: 363-372.

56. Schaufelberger SA, Rosselli M, Barchiesi F. 2-Methoxyestradiol,an endogenous 17ß-estradiol metabolite, inhibits microglial proliferation and activation via an estrogen receptor-independent mechanism. Am J Physiol Endocrinol Metab. 2016; 310: E313-E322.

57. Ishihara $Y$, Itoh K, Ishida A. Selective estrogen-receptor modulators suppress microglial activation and neuronal cell deathvia an estrogen receptor-dependent pathway. J Steroid Biochem Mol Biol. 2015; 145: 85-93.

58. Wu SY, Chen YW, Tsai SF. Estrogen ameliorates microglial activation by inhibiting the Kir2.1 inwardrectifier K(+) channel. Sci Rep. 2016; 6: 1-14.

59. Barrientos R, Frank M, Watkins L. Memory impairments in healthy aging: Role of aging-induced microglial sensitization. Aging Dis. 2010; 1: 212-231.

60. Palin K, Cunningham C, Forse P. Systemic inflammation switches the inflammatory cytokine profile in CNS wallerian degeneration. Neurobiol Dis. 2008; 30: 19-29.

61. Fenn AM, Gensel JC, Huang Y. Immune activation promotes depression 1 month after diffuse brain injury: a role for primedmicroglia. Biol Psychiatry. 2014; 76: 575-84.

62. Khasnavis S, Ghosh A, Roy A. Castration induces Parkinson disease pathologies in young male mice via inducible nitric-oxidesynthase. J Biol Chem. 2013; 288: 20843-20855.

63. Kreisel T, Frank MG, Licht T. Dynamic microglial alterations underlie stress-induced depressive-like behavior and suppressed neurogenesis. Mol Psychiatry. 2014; 19: 699-709.

64. Tremblay ME, Stevens B, Sierra A. The role of microglia in the healthy brain. J Neurosci. 2011; 31: 16064-16069.

65. Kettenmann H, Hanisch UK, Noda M. Physiology of microglia. Physiol Rev. 2011; 91: 461-553.

66. Ménard C, Hodesm GE, Russo SJ. Pathogenesis of depression: Insights from human and rodent studies. Neuroscience. 2016; 321: 138-162.

67. Fernández-Guasti A, Vega-Matuszczyk J, Larsson K. Synergistic action of estradiol, progesterone and testosterone on rat proceptive behavior. Physiol Behav. 1991; 50 :1007-1011.

68. Fujimoto N, Kanno J. Increase in prostate stem cell antigen expression in prostatic hyperplasia induced by testosterone and 17ß-estradiol in C57BL mice. J Steroid Biochem Mol Biol. 2016; 158: 5662. 
69. Ewing LL, Desjardins C, Irby DC. Synergistic interaction of testosterone and oestradiol inhibits spermatogenesis in rats. Nature. 1977; 269: 409-411.

70. Miclea A, Miclea M, Pistor M. Intramuscular testosterone supplementation ameliorates depression in hypogonadal men: a retrospective study in an outpatient department. 2018; 12: 254-263.

71. Gillespie CF, Nemeroff CB. Hypercortisolemia and depression. Psychosom Med. 2005; 67: 26-28.

72. Walker FR, Beynon SB, Jones KA. Dynamic structural remodelling of microglia in health and disease: a review of the models, the signals and the mechanisms. Brain Behav Immun. 2014; 37: 1-14.

73. Kim KH, Son SM, Mook-Jung I. Contributions of microglia to structural synaptic plasticity. J Exp Neurosci. 2013; 7: 85-91.

74. Brunoni AR, Machado-Vieira R, Zarate CA. BDNF plasma levels after antidepressant treatment with sertraline and transcranial direct current stimulation: results from a factorial, randomized, shamcontrolled trial. Eur Neuropsychopharmacol. 2014; 24: 1144-1151.

\section{Figures}
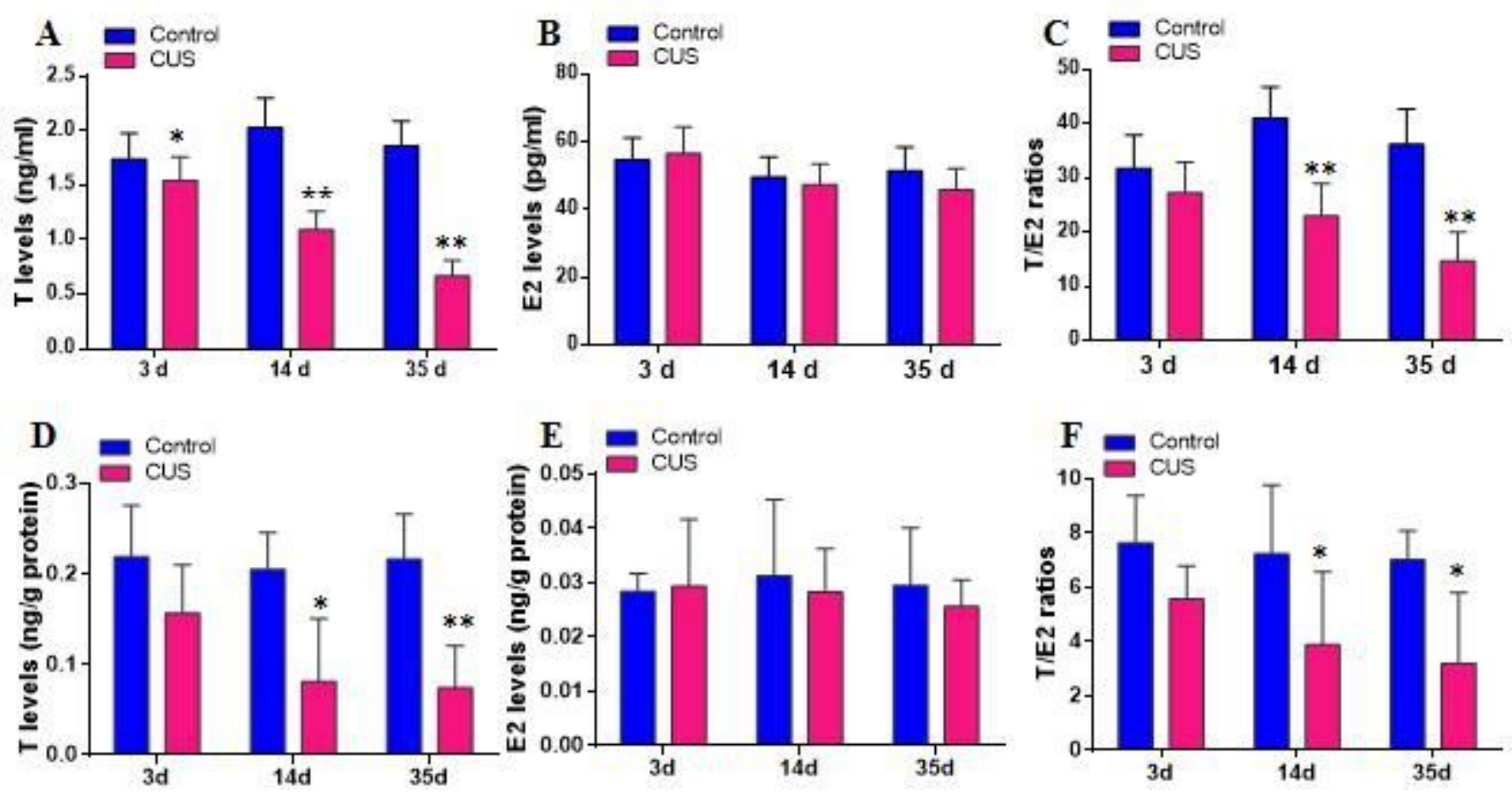

\section{Figure 1}

Depression-associated changes in testosterone and estradiol levels as well as testosterone/estradiol ratio. (A-C) LC-MS/MS analysis of (A) testosterone level, $(B)$ estradiol level and (C) testosterone/estradiol ratio in serum from mice at 3-35 days after exposure to chronic unpredictable stress (CUS; $\mathrm{n}=6$ per group). (D-F) LC-MS/MS analysis of (D) testosterone level, (E) estradiol level and (F) testosterone/estradiol ratio in brain tissue from mice at 3-35 days after exposure to CUS ( $n=6$ per group). ${ }^{\star} P<0.05,{ }^{\star *} P<0.01$ compared to control group. 

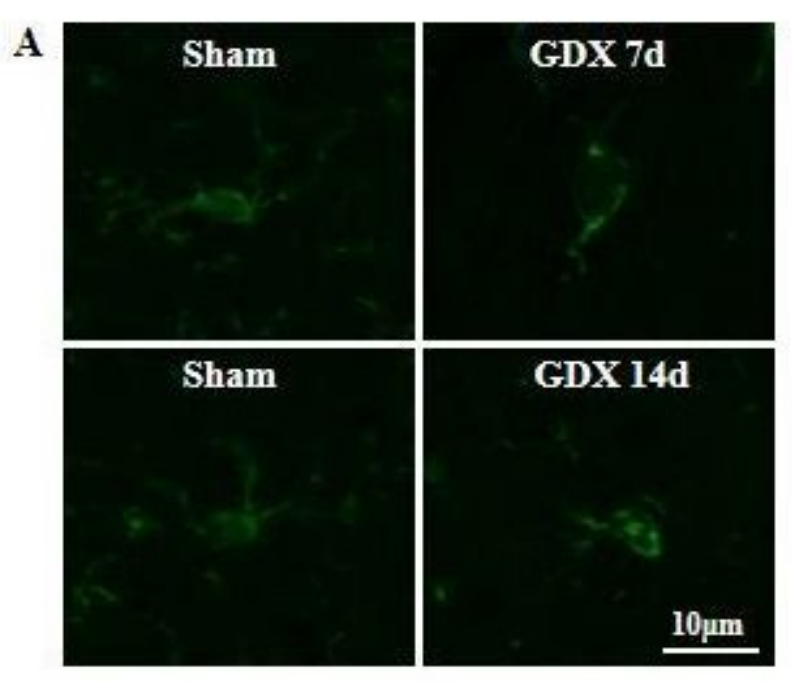

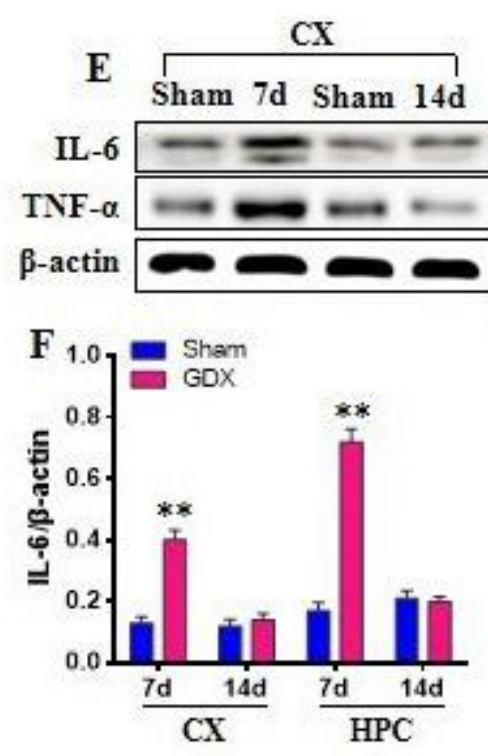

HPC
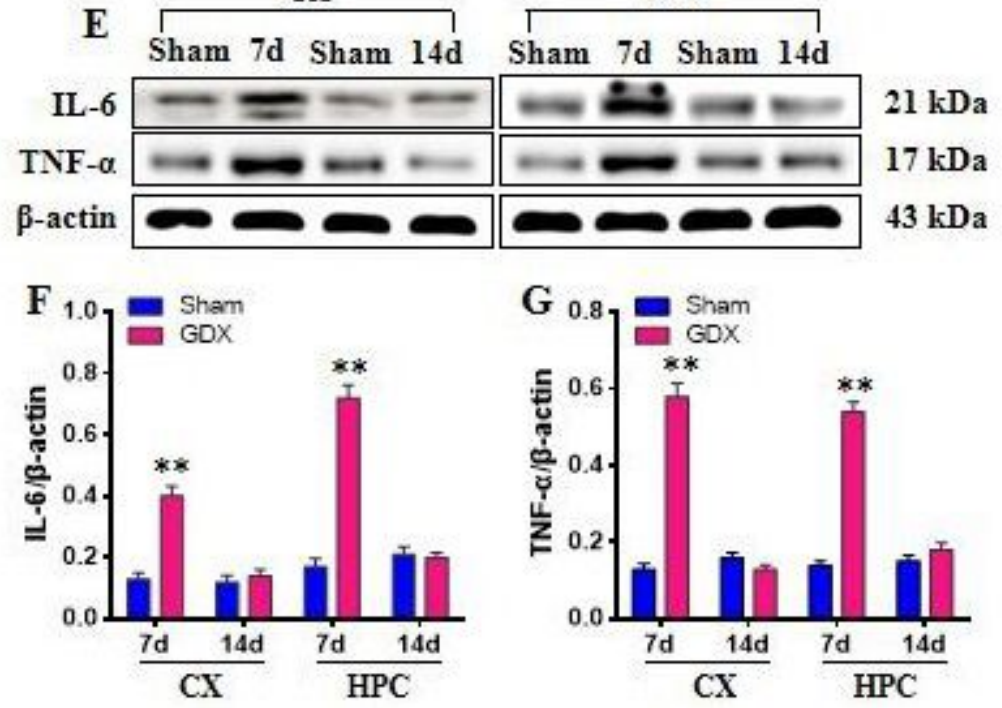
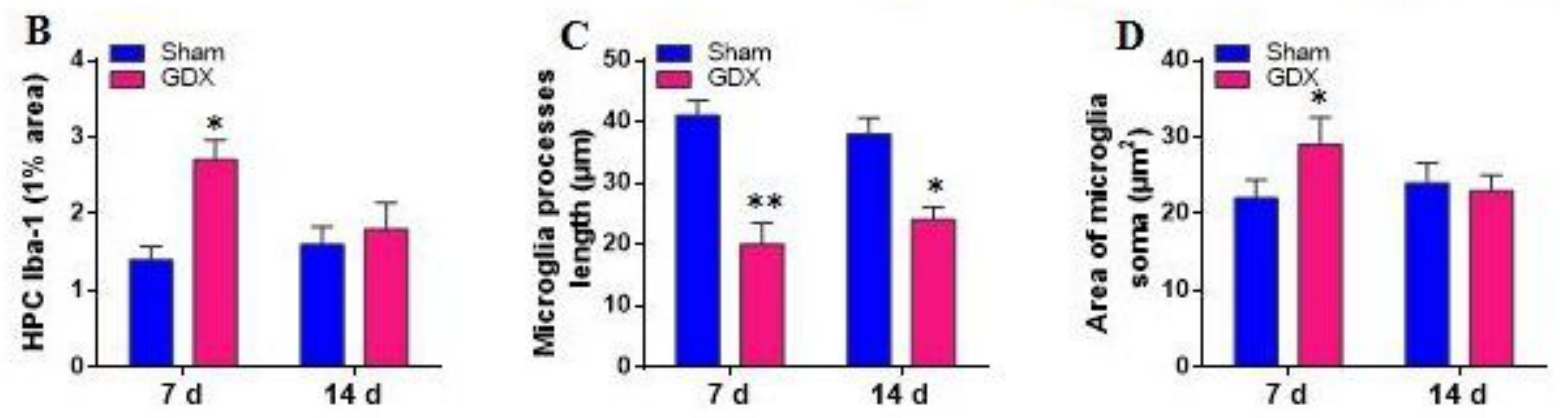

Figure 2

Effects of short-term gonadal hormone deficiency on microglia activation and inflammatory response in the brain. (A) Representative micrographs of Iba-1-labeled microglia in sham-operated or GDX mice at 7 or 14 days after surgery. (B-D) Indices of microglial activation at 7 or 14 days after gonadectomy or sham surgery: (B) Proportion of area staining for Iba-1, (C) microglial process length and (D) soma size. (E) Effects of sex hormone deficiency on levels of IL- 6 and TNF-a in the cortex and hippocampus on days 7 and 14 ( $n=4$ per group). (F) Comparison of IL-6 levels between sham-operated and GDX mice on days 7 and 14 following surgery. $(G)$ Comparison of TNF-a levels between sham-operated and GDX mice on days 7 and 14 following surgery. ${ }^{\star} P<0.05,{ }^{\star} P<0.01$ compared to the sham group. 

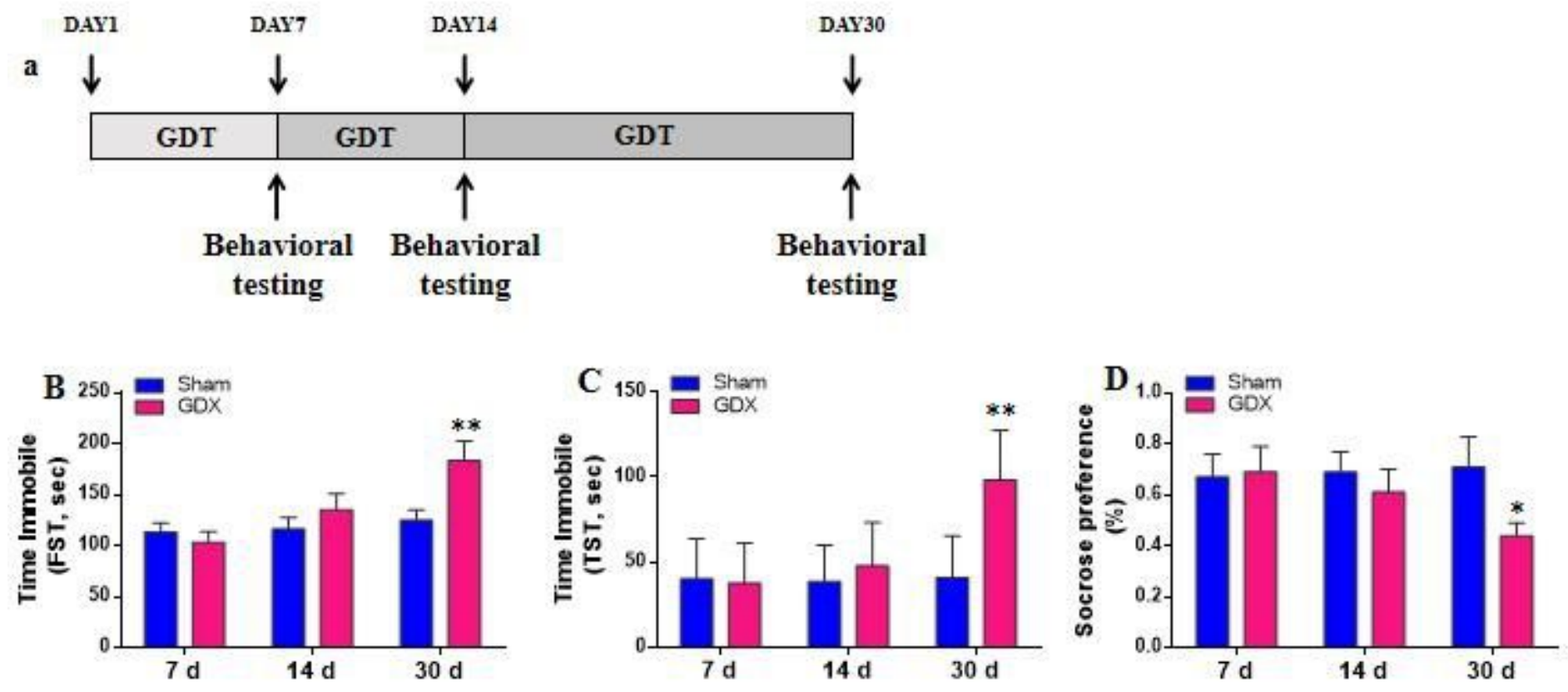

Figure 3

Effects of long-term gonadal hormone deficiency on the development of depressive-like behaviors. (A) Experimental paradigm of gonadectomy (7, 14 or 30 days) exposure and behaviors testing in shamoperated and gonadectomized (GDX) mice. (B-D) Effects of gonadectomy on depressive behavior, measured using a (B) forced swim test (FST), (C) tail suspension test (TST), and (D) sucrose preference test (SPT). ${ }^{\star} P<0.05,{ }^{*} P<0.01$ compared to the sham group. 

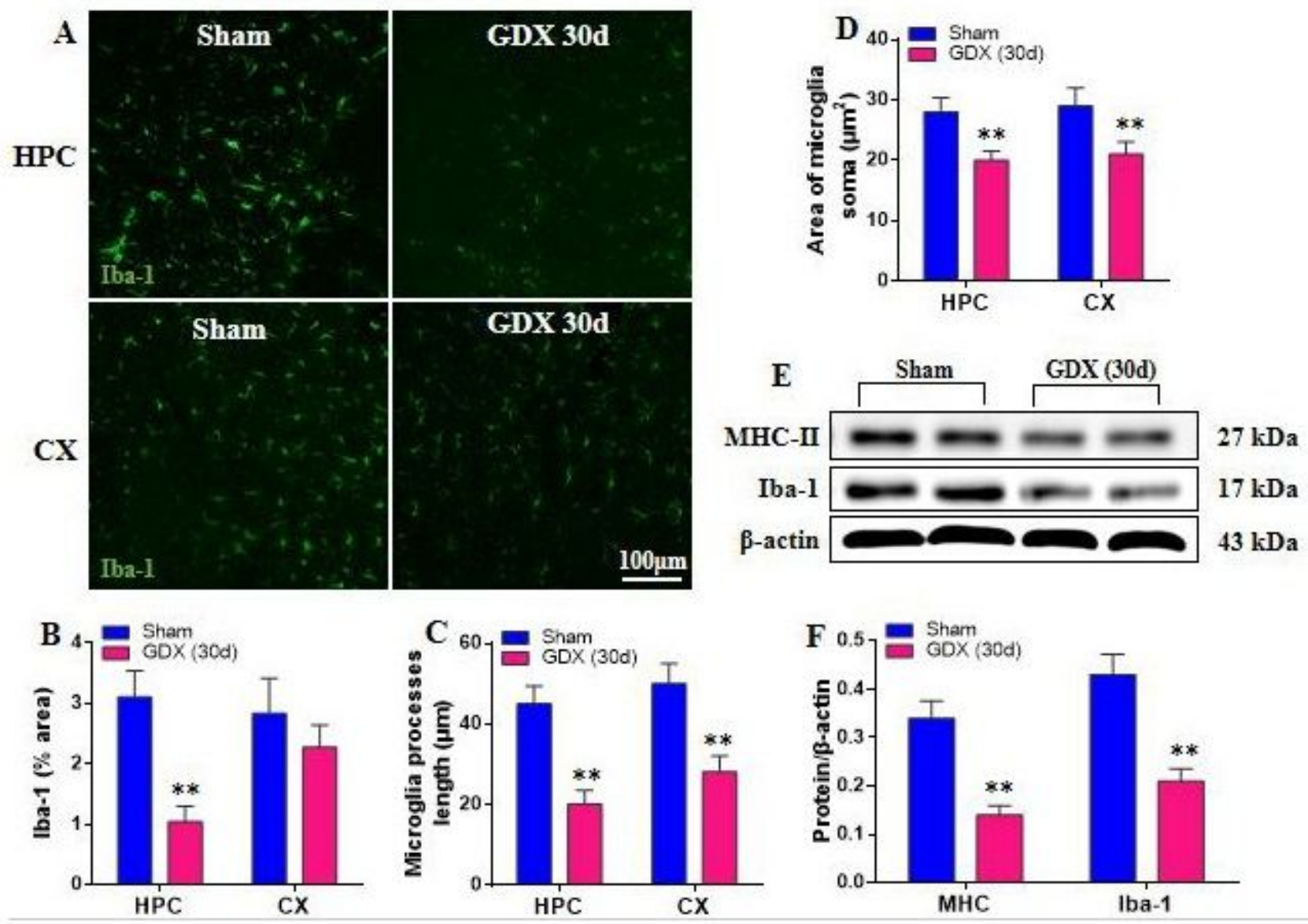

\section{Figure 4}

Effects of long-term gonadal hormone deficiency on microglia decline. (A) Representative micrograph of Iba-1-labeled microglia in hippocampus (HPC) and cortex (CX) of sham-operated and gonadectomized (GDX) mice. (B) Comparison of proportional area for Iba-1 staining in HPC and CX between shamoperated and GDX mice ( $n=4$ per group). (C) Effects of GDX on length of microglial processes. (D) Effects of GDX on soma area of microglia in HPC and CX. (E) Comparison of MHC-II and Iba-1 levels in hippocampal microglia between sham-operated and GDX mice. (F) Comparison of microglial activation markers in HPC between sham-operated and GDX mice. Tests were performed on days 7, 14, and 30 following gonadectomy. ${ }^{*} P<0.01$ compared to the sham group. 


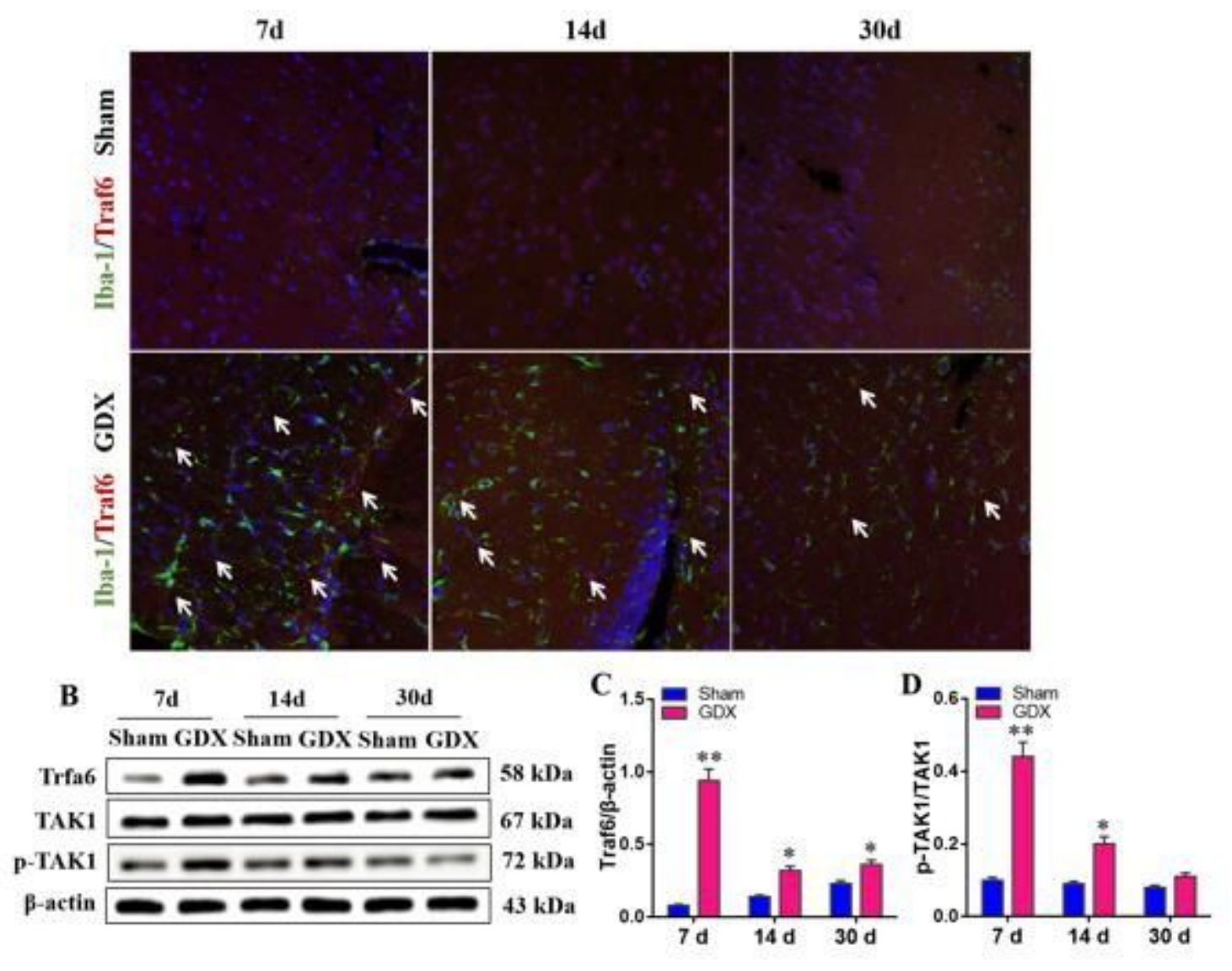

\section{Figure 5}

Effects of gonadal hormone deficiency on Traf6/TAK1 signaling pathway. (A) Representative micrograph of Iba-1/Traf6-doubling labeled microglia in hippocampus (HPC) of sham-operated and gonadectomized (GDX) mice. (B) Representative Western blotting results for Traf6, TAK1 and p-TAK1 proteins in hippocampal microglia. (C) Comparison of Traf6 expression in hippocampal microglia between shamoperated and GDX mice. (D) Comparison of TAK1 phosphorylation in hippocampal microglia among sham-operated and GDX mice. Tests were performed on days 7, 14, and 30 following gonadectomy. ${ }^{*}<$ $0.05,{ }^{\star *} P<0.01$ compared to the sham group. 


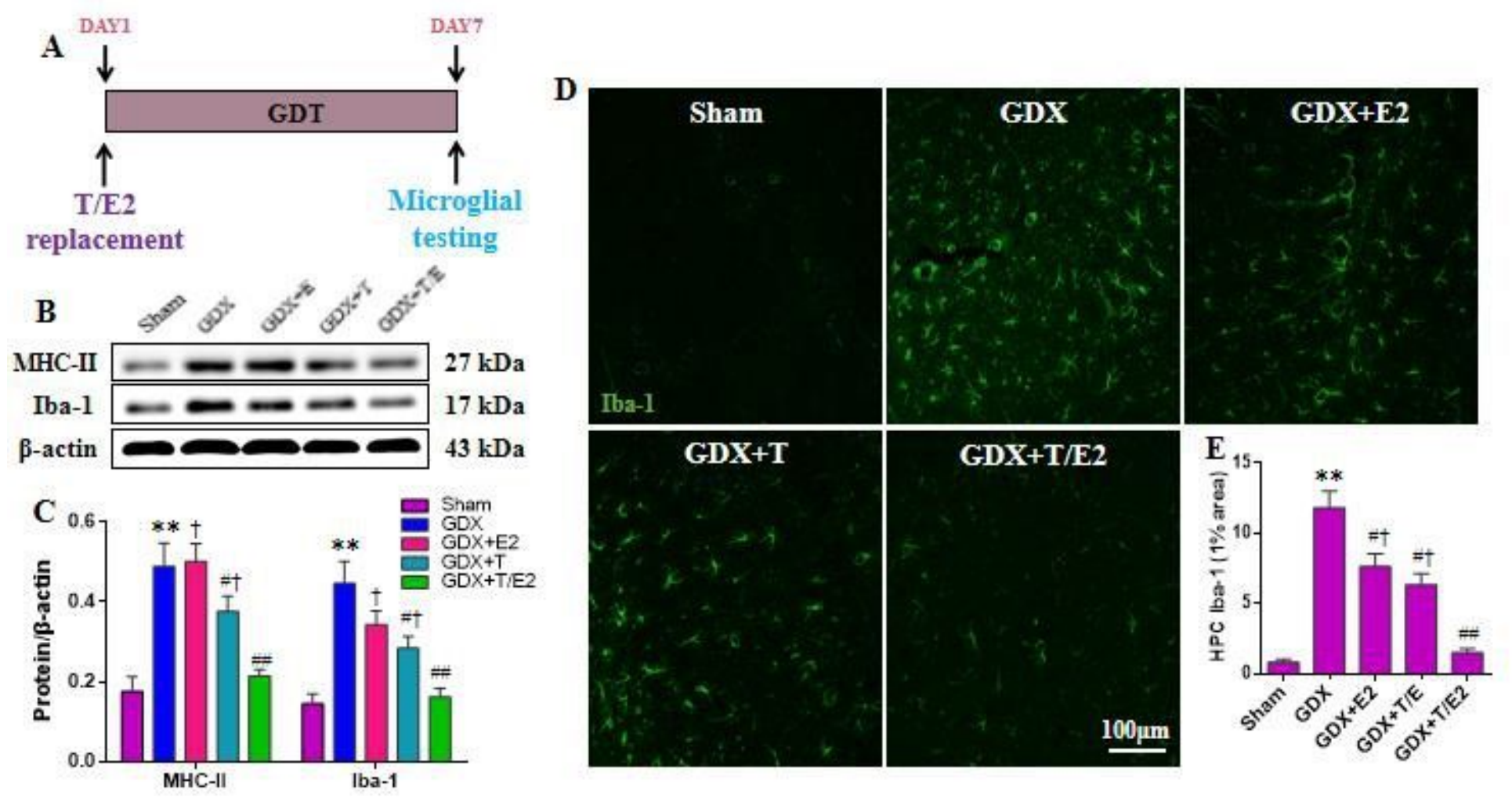

Figure 6

Effects of sex hormone replacement on gonadectomy-induced changes in microglia activation. Shamoperated or gonadectomized (GDX) mice were treated with hormone replacement in the form estradiol alone (E), testosterone $(\mathrm{T})$ alone or the mixture of testosterone and estradiol in a 35:1 ratio (T/E) for 7 days. (A) Experimental paradigm of gonadectomy exposure and T/E replacement for 7 days in shamoperated and gonadectomized (GDX) mice. (B) Representative Western Blotting results for microglial activation markers in hippocampus. (C) Effects of 1-week hormone replacement on levels of MHC-II and Iba-1. (D) Representative micrograph of Iba-1-labeled microglia in hippocampus (HPC) of sham-operated and gonadectomized (GDX) mice. (E) Comparison of proportional area for lba-1 staining in HPC between sham-operated and GDX mice after treatment with sex hormone ( $\mathrm{n}=4$ per group). ${ }^{*} P<0.05,{ }^{*} P<0.01$ compared with the sham-operated group; ${ }^{\#} P<0.05$, ${ }^{\# \#} P<0.01$ compared with the GDX group; ${ }^{\dagger} P<0.05$ compared with the GDX+T/E group. 


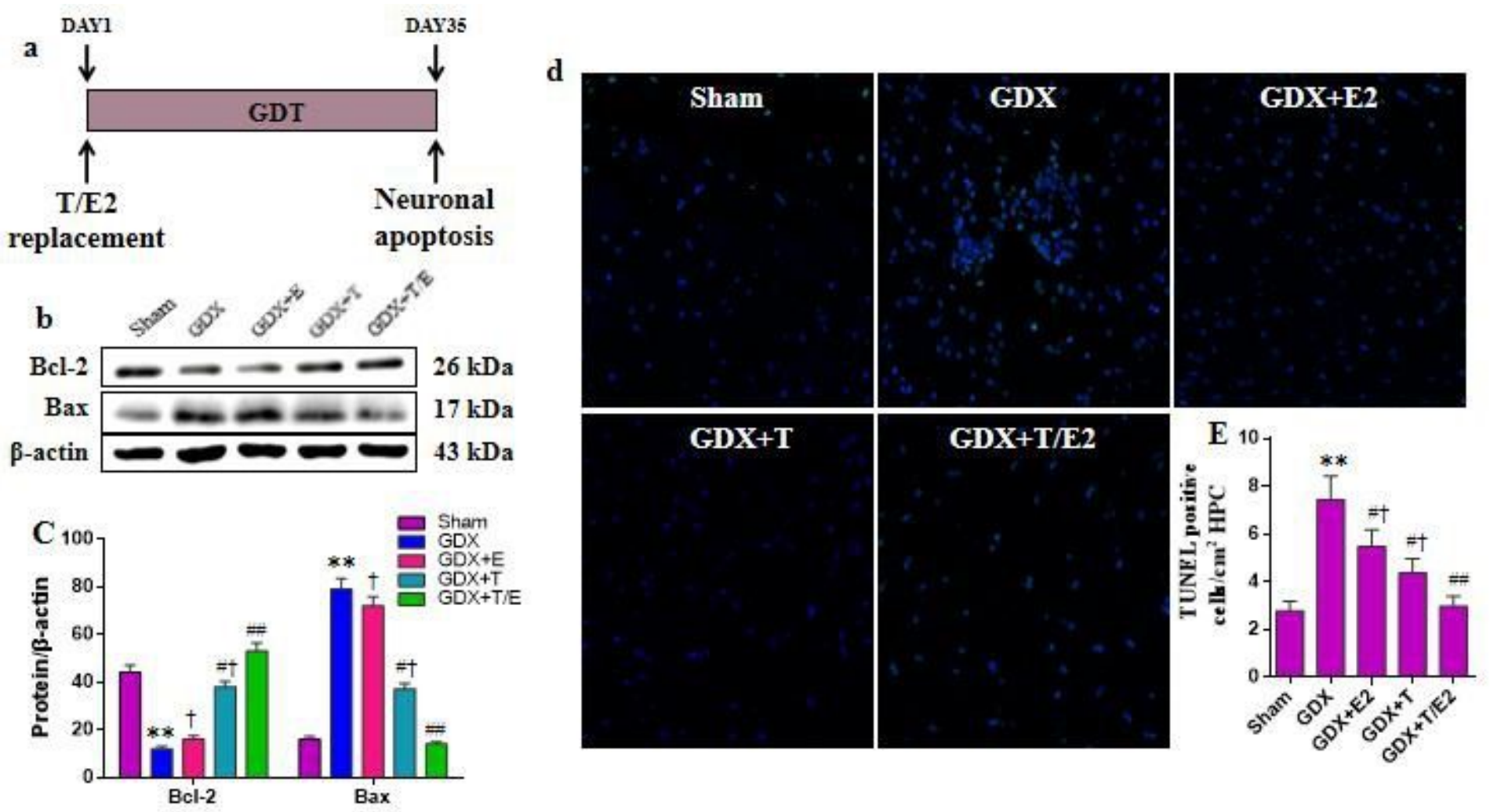

Figure 7

Effects of sex hormone replacement on gonadectomy-induced changes in neuronal apoptosis. (A) Experimental paradigm of gonadectomy exposure and T/E replacement for 30 days in sham-operated and gonadectomized (GDX) mice. (B) Representative Western blotting results for apoptotic proteins in hippocampus. (C) Effects of 5-week hormone replacement on levels of Bcl-2 and Bax. (D) Effects of 5week hormone replacement on the number of TUNEL-stained cells in the hippocampus. (E) Comparison of TUNEL positive cells in hippocampus after treatment with sex hormone ( $\mathrm{n}=4$ per group). ${ }^{* \star} P<0.01$ compared with the sham-operated group; ${ }^{\#} P<0.05$, ${ }^{\# \#} P<0.01$ compared with the GDX group; ${ }^{\dagger} P<0.05$ compared with the GDX+T/E group. 

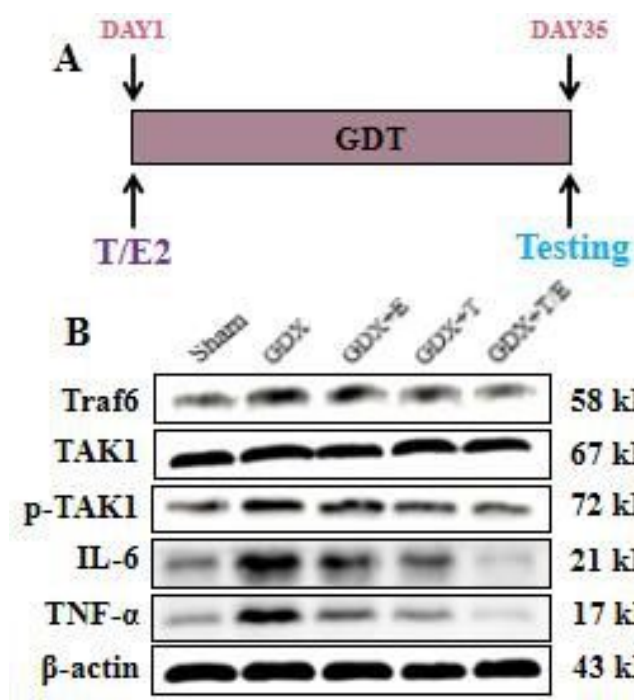

$58 \mathrm{kDa}$

$67 \mathrm{kDa}$

$72 \mathrm{kDa}$
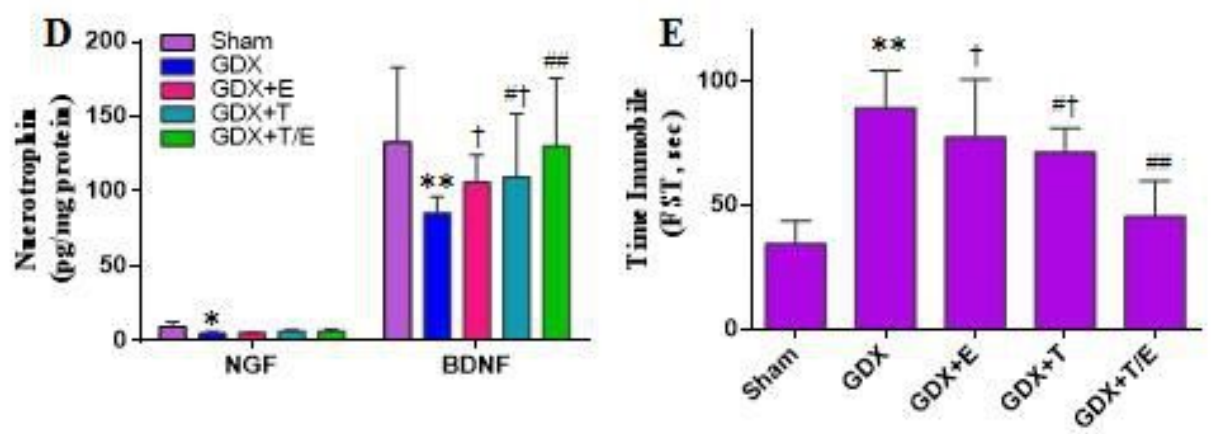

$21 \mathrm{kDa}$
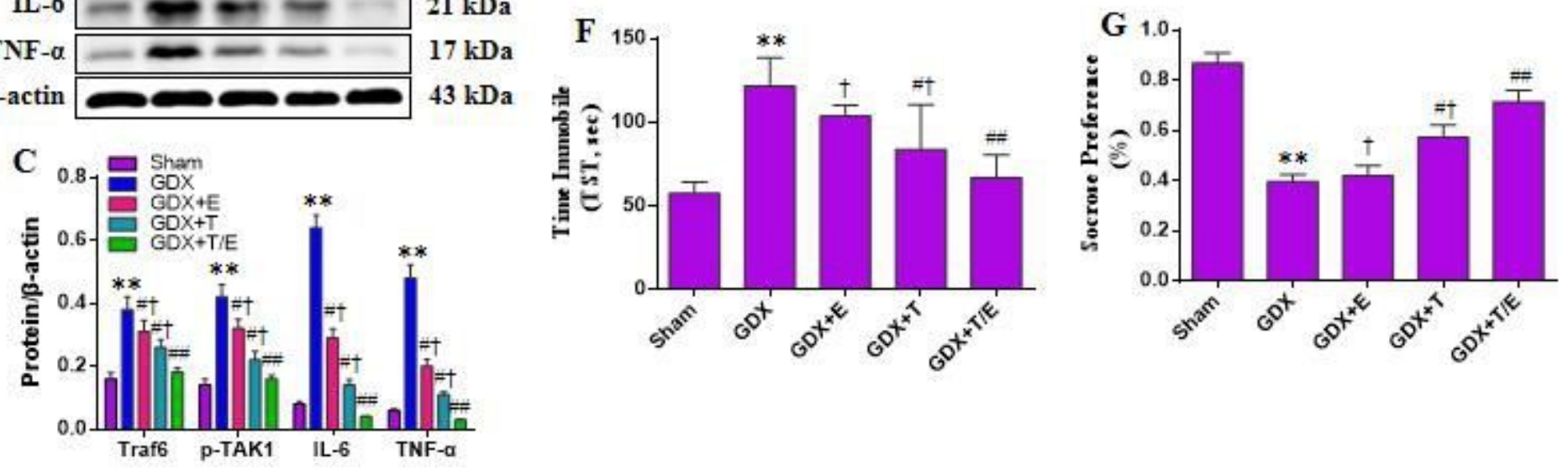

$17 \mathrm{kDa}$ $43 \mathrm{kDa}$

\section{Figure 8}

Effects of sex hormone replacement on gonadectomy-induced changes in depressive behaviors, neuroinflammation and neurogenesis. (A) Experimental paradigm of gonadectomy exposure and T/E replacement for 30 days in sham-operated and gonadectomized (GDX) mice. (B) Representative Western Blotting results for Traf6-mediated inflammatory cytokines in hippocampal microglia. (C) Effects of 5week hormone replacement on levels of Traf6, p-TAK1, IL-6 and TNF-a. (D) Effects of 5-week hormone replacement on levels of neurotrophin. (E-G) Effects of 5-week hormone replacement on performance in the (E) FST, (F) TST and (G) sucrose preference test. $* * P<0.01$ compared with the sham-operated group; ${ }^{\#} P<0.05,{ }^{\#} P<0.01$ compared with the GDX group; ${ }^{\dagger} P<0.05$ compared with the GDX+T/E group.

\section{Image not available with this version}

\section{Figure 9}

A model for how gonadal hormone deficiency may contribute to microglial activation. In response to gonadal hormone deficiency insults, the Traf6 expression levels in microglia are upregulated. Traf6 then 
induces phosphorylation and activation of TAK1. TAK1 further activates nuclear factor-KB (NF-KB) signaling pathway, leading to enhanced inflammatory response in microglia. The effects of Traf6/TAK1 signaling cascade results in microglial priming. Whereas testosterone and estradiol could inhibit the expression and ubiquitination of Traf6 protein via AR/ERa-mediated pathways, and further suppress the microglial activation. 